\title{
The Central Sensitization Inventory validated and adapted for a Brazilian population: psychometric properties and its relationship with brain-derived neurotrophic factor
}

This article was published in the following Dove Press journal:

Journal of Pain Research

I September 2017

Number of times this article has been viewed

\author{
Wolnei Caumo ${ }^{1-4}$ \\ Luciana C Antunes' \\ Jéssica Lorenzzi Elkfury' \\ Evelyn G Herbstrith ${ }^{5}$ \\ Raquel Busanello Sipmann ${ }^{6}$ \\ Andressa Souza ${ }^{7}$ \\ Iraci LS Torres ${ }^{1,8}$ \\ Vinicius Souza dos Santos' \\ Randy Neblett ${ }^{9}$ \\ 'Postgraduate Program in Medical \\ Sciences, School of Medicine, \\ 2Pain and Palliative Care Service, \\ Hospital de Clínicas de Porto \\ Alegre, ${ }^{3}$ Laboratory of Pain and \\ Neuromodulation, ${ }^{4}$ Surgery \\ Department, School of Medicine, \\ Universidade Federal do Rio Grande \\ do Sul, Porto Alegre, ${ }^{5} \mathrm{School}$ of \\ Psychology, Pontifícia Universidade \\ Católica do Rio Grande do Sul, \\ ${ }^{6}$ School of Medicine, Universidade \\ Federal do Rio Grande do Sul, \\ ${ }^{7}$ Postgraduate Program in Health \\ and Human Development, La \\ Salle University Center, Canoas, \\ ${ }^{8}$ Pharmacology Department, Instituto \\ de Ciências Básicas da Saúde, \\ Universidade Federal do Rio Grande \\ do Sul, Porto Alegre, Brazil; ' ${ }^{\text {PRIDE }}$ \\ Research Foundation, Dallas, TX, USA
}

Correspondence: Wolnei Caumo Surgery Department, School of Medicine, Hospital de Clínicas de Porto Alegre, Universidade Federal do Rio Grande do Sul, 2350 Ramiro Barcelos Street, Porto Alegre, RS 90035-003, Brazil Email wcaumo@hcpa.edu.br
Objectives: The primary aim was to assess the psychometric properties (including internal consistency, construct validity, reproducibility, and factor structure) of the Central Sensitization Inventory (CSI), adapted and validated for a Brazilian population (CSI-BP). Additionally, we evaluated the relationship between the CSI-BP and the serum brain-derived neurotrophic factor (BDNF) and determined if the symptoms elicited by the CSI-BP discriminate between subjects who do/do not respond to the conditioned pain modulation (CPM) task, as assessed by change in numeric pain scale $(0-10)$ score.

Patients and methods: A cross-sectional study was conducted in a pain clinic in a tertiary teaching hospital. A total of 222 adults with chronic musculoskeletal pain and 63 healthy control subjects completed the CSI-BP and the Brazilian Portuguese pain-catastrophizing scale (BPPCS). A team of experts translated the CSI according to the international guidelines. Test-retest, item analysis, convergent validity, and factor analysis were performed. Later, a random subsample $(\mathrm{n}=77)$ was used to correlate the CSI-BP adjusted index with change in numeric pain-scale score during the CPM task and a BDNF blood sample.

Results: The CSI-BP presented strong psychometric properties (test-retest reliability 0.91, Cronbach's $\alpha=0.91$ ). Confirmatory factor analysis yielded a four-factor structure, supporting the original English version. The CSI-BP adjusted index showed moderate positive correlation with the BP-PCS, and classified more than $80 \%$ of patients correctly vs healthy controls. Serum BDNF levels explained $27 \%$ of the variation in the CSI-BP adjusted index. Subjects with impairment in the descending modulatory system had higher CSI-BP adjusted index scores than subjects who responded normally to the CPM task: 49.35 (12.1) vs $39.5(12.33)$, respectively $(P<0.05)$. Conclusion: The CSI-BP was found to be a psychometrically strong and reliable instrument, with primary evidence of validity. Higher scores on the CSI-BP were correlated positively with serum BDNF and with greater dysfunction of the descending pain-modulatory system.

Keywords: confirmatory factor analysis, cross-cultural adaptation, conditioned pain modulation, serum brain-derived neurotrophic factor, central sensitization, chronic pain

\section{Introduction}

Chronic pain is one of the most prominent causes of disability worldwide. A meta-analysis of population studies showed an estimated prevalence of widespread chronic pain of $14.2 \% .{ }^{1}$ Another meta-analysis found a predominance of chronic musculoskeletal pain in elderly Brazilians of $14.1 \%-85.5 \% .^{2}$ It is often associated with other clinical symptoms, including fatigue, poor sleep, cognitive deficits, headaches, depression, and anxiety. ${ }^{3}$ These symptoms are frequently related to central sensitization (CS), 
identified in many disorders. CS has overlapping symptoms in a spectrum of structural pathology, from those with persistent nociception, eg, osteoarthritis (OA), to those without physical tissue damage, such as fibromyalgia (FM) and myofascial pain syndrome (MPS). ${ }^{4}$ Yunus proposed the term "central sensitivity syndrome" (CSS) to categorize inorganic painrelated disorders with overlapping symptom dimensions, with CS being the common etiology. ${ }^{5}$

$\mathrm{CS}$ and associated symptoms are a consequence of changes in the central nervous system (CNS) that amplify the response to nociceptive inputs and fail to suppress noise signals, rather than just reflecting the presence of information in a set of sensory fibers. ${ }^{6,7}$ This syndrome comprises impaired functioning of neurons and circuits in nociceptive pathways, increased membrane excitability and synaptic efficacy, and reduced inhibition. ${ }^{8}$ Sensitized neurons of the spinal dorsal horn exhibit increased spontaneous activity, reduction in threshold for activation, and an enlargement of their receptive fields. ${ }^{8}$ Furthermore, CS-related changes have been found in the microglia, astrocytes, gap junctions, and gene transcription, contributing to the maintenance of the general state of excitation. Since the release of brain-derived neurotrophic factor (BDNF) is part of spinal microglial activation, it contributes to both induction and maintenance of CS. ${ }^{9}$ These impairments in pain circuits lead to the hypersensitivity observed in many chronic pain conditions. However, few tools have been available to help clinicians to identify when a patient presents symptoms that may be related to CS or may indicate the presence of CSS. The CS inventory (CSI) is a self-report questionnaire of health symptoms designed as an easy-to-administer screener for patients who are at high risk of having CS or to assess CS-related symptoms. The use of the CSI has also been recommended as one component of an algorithm to help classify chronic pain patients ${ }^{10}$ and lowback pain patients ${ }^{11}$ with CS and to help differentiate them from patients with primary neuropathic and nociceptive pain. Most recently, five CSI severity levels have been proposed to aid clinical interpretation and evaluation of treatment responsiveness. ${ }^{12}$ The CSI is currently available in English, Central American Spanish, and Dutch ${ }^{13}$ and is being translated and validated in other European and Asian languages. ${ }^{14-18}$

Considering that chronic pain is highly prevalent across cultures, the recognition that it is often related to CS necessitates having a reliable instrument to assess CS-related symptoms and to analyze the relationship of its score with neuroplasticity biomarkers. The primary aim was to assess the psychometric properties (including internal consistency, construct validity, reproducibility, and factor structure) of the CSI adapted and validated for a Brazilian population (CSI-BP). Additionally, we evaluated the relationship of the CSI-BP with serum BDNF and determined the ability of CSI-BP symptoms to discriminate between subjects who do/do not respond to a conditioned pain modulation (CPM) task, as assessed by change in a numeric pain scale (0-10).

Our hypothesis was that the degree of symptoms indexed by total CSI-BP scores would identify the severity of CS, according to the physiopathology of pain condition, such as FM, MPS, chronic tension-type headache (CTTH), OA, and pain-free healthy control subjects. Also, we hypothesized that CSI-BP scores would be correlated positively with blood levels of BDNF and would discriminate between subjects who $\mathrm{did} / \mathrm{did}$ not respond to a CPM task.

\section{Patients and methods}

The protocol was reviewed and approved by the ethics committee board of the Hospital de Clínicas de Porto Alegre (protocol 10-0555). All subjects gave their written informed consent before participation.

\section{Phase I: translation, synthesis, and back-translation}

The procedures for the translation and adaptation of the CSI to Brazilian Portuguese and assessment of the semantics and conceptual content of each item (content validity) were conducted through the Delphi method (see Supplementary material).

\section{Phase II: pretesting CSI-BP in pilot version}

Thirty medical school employees (15 women) who work with chronic pain patients volunteered to evaluate the meaning of the translated items and the layout of the "prefinal" version of the CSI-BP. Also, 20 females with FM who had volunteered to evaluate the meaning of the translated CSI-BP questions were included and they completed the CSI-BP twice, and the interval for test-retest reliability of the CSI-BP was 15 days. The assessment regarding the comprehension of the items and all feedback from these subjects were evaluated by a translation workgroup (to assess face validity) (see Supplementary material).

\section{Phase III: assessment of psychometric properties and the validity of the final version of the CSI-BP \\ Subjects}

A total of 667 chronic pain subjects and 86 healthy control subjects were assessed for eligibility. They were recruited 
from local community care units, an institutional chronic pain clinic, by referrals from other hospital units, and by phone and newspaper. The recruitment process was done in conjunction with other randomized controlled trials (RCTs) run at the Hospital de Clínicas de Porto Alegre. These RCTs, registered at https://clinicaltrials.gov, included studies with the pain-related disorders FM (NCT02041455, NCT01804097), MPS (NCT01964729), CTTH (NCT01954277), and OA (NCT01747070, NCT01855958) (Figure 1). An experienced physician trained in chronic pain assessment and treatment confirmed all diagnoses in the RCTs using standardized evaluation criteria. For the present CSI-BP study, 222 patients were determined to be eligible and agreed to participate: 73 with FM, 65 with MPS, 53 with CTTH, and 31 with OA.

Diagnoses for patients in the six RCTs were performed using standard assessment protocols. The diagnostic criteria for MPS included regional pain, normal neurologic examination, the presence of trigger points, taut bands, and tender points, and pain characterized as "dull", "achy", or "deep". OA diagnoses were made according to the clinical and radiographic criteria of the American College of Rheumatology. Also, all OA patients completed the Western Ontario and McMaster universities OA index, a validated measure of OA-related patient-reported symptoms and perceived disability. ${ }^{19}$ CTTH was diagnosed using the 2004 International Headache Society criteria. ${ }^{20} \mathrm{FM}$ was diagnosed according to the American College of Rheumatology criteria for FM. ${ }^{21}$ In all six RCTs, patients were included if they had experienced pain scored as visual analog scale (VAS) $40 \mathrm{~mm}$ (ie, moderate or severe pain). Additionally, the pain needed to be associated with disability, as assessed by an affirmative answer to dichotomous questions (yes/no) of a structured questionnaire. Queries inquired if their pain had interfered with work, enjoyable activities, responsibilities at home, relationships, personal goals, thinking clearly, problem solving, concentration, or recall. We excluded patients with a rheumatic or neurologic

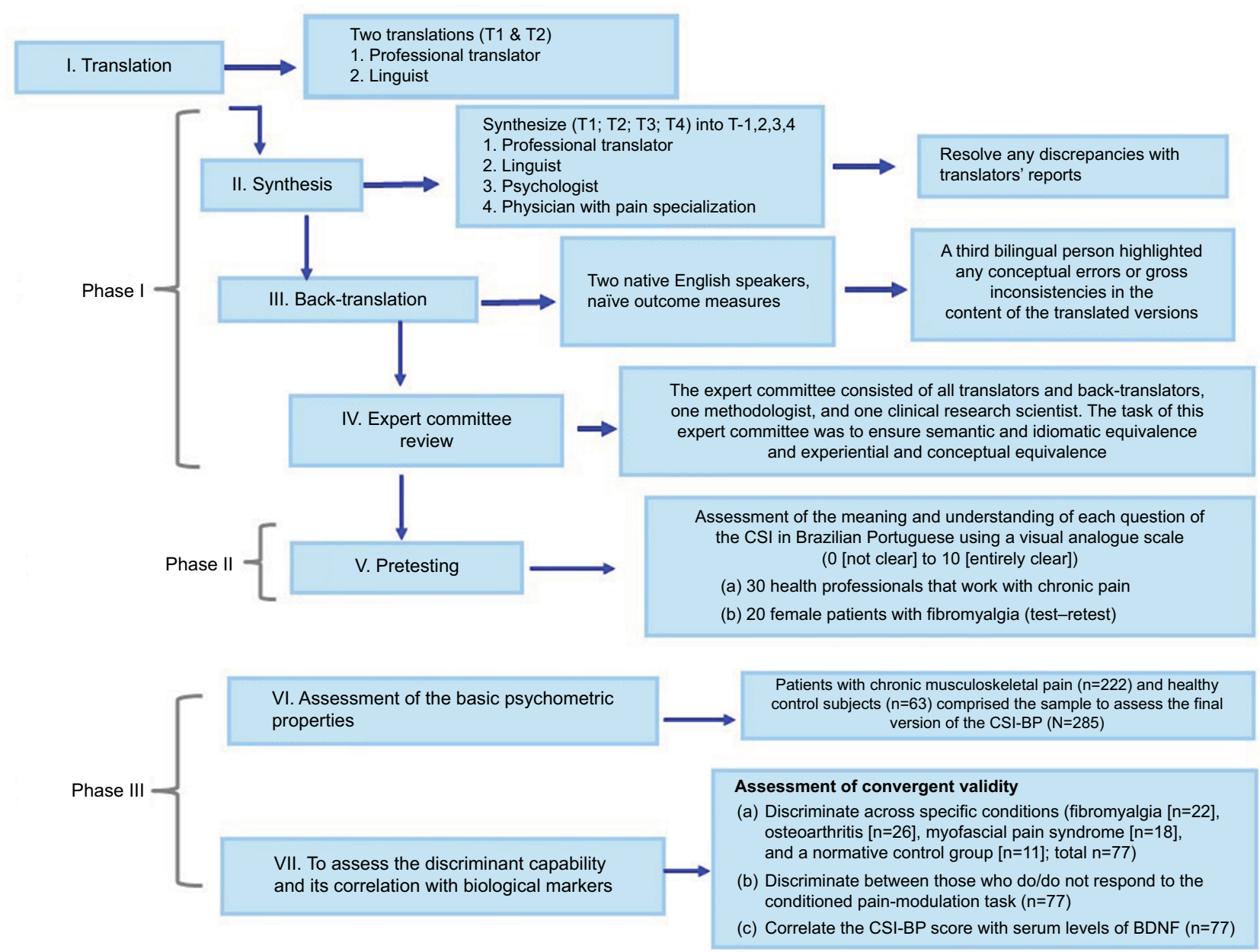

Figure I Flow of the multiple standardized phases of the study.

Abbreviations: BDNF, brain-derived neurotrophic factor; CSI, Central Sensitization Inventory; CSI-BP, Central Sensitization Inventory - Brazilian population. 
condition, such as stroke or Parkinson's disease, surgery on the affected areas in the prior 6 months, habitual use of corticosteroids, or other uncompensated chronic pathologies. We excluded patients with any malignancy diagnosis, with HIV, or if they were illiterate.

Healthy controls were recruited from the general population using public postings as well. Of the 86 potential healthy control subjects, 63 were eligible and agreed to participate. A standard screening questionnaire was performed to assess inclusion criteria. Eligible healthy control subjects had to be free of any acute or chronic pain, as well as any rheumatologic, psychiatric, or neurological disorders, and without recent use of analgesics, corticosteroids, or medications with known effects on the CNS. Also, healthy control volunteers were not included if they reported abuse of alcohol or psychotropic substances in the 6 months prior to the screening. Unlike the patient sample, none of the control volunteers underwent a physical examination.

We selected 66 patients among 159 patients with chronic pain (OA 31, FM 77, and MPS 65) and 11 subjects among 63 in the healthy group. The randomization procedure was conducted within each group of pathologies using a simple random method. This subsample included 26 OA, 22 FM, 18 MPS, and 11 healthy control subjects (pain-free). A physician with more than 15 years of experience in a pain clinic and highly skilled at diagnosing chronic pain conditions reexamined patients and then reconfirmed their diagnosis. CSI-BP scores obtained in this subsample were used for correlations with two biological markers of CS: CPM task and BDNF blood sample. Also, patients answered questions during a structured interview, and a blood sample was collected.

\section{Self-report variables}

After signing informed consent, subjects completed a sociodemographic questionnaire that assessed variables including age, gender, work status due to pain, marital status, and education. In addition to the CSI, all subjects completed the Brazilian Portuguese pain-catastrophizing scale (BPPCS). ${ }^{22}$ This questionnaire consists of 13 items evaluating self-reported catastrophizing thoughts, feelings, and behaviors when one is in pain. Total scores, ranging from 0-52, are computed by summing all items on the questionnaire..$^{22,23}$ The BP-PCS evaluates aspects of pain that are related to CSI items, and because of this it was chosen to help the assessment of CSI-BP convergent validity.

In addition to the sociodemographic questionnaire and BP-PCS, patients from three subsample groups (FM, OA, and MPS) who participated in the biological marker section of the study also completed two additional self-report measures. Pain severity was assessed by visual analogue scale (VAS). VAS results were converted to 0 (no pain) to 10 (worst possible). Depressive symptoms were measured with the Beck Depression Inventory II, adapted to Brazilian Portuguese, ${ }^{24}$ which is an instrument recommended by the Initiative on Methods, Measurement, and Pain Assessment in Clinical Trials because of its excellent psychometric properties and extensive use in clinical pain research.

\section{CS-related symptom variables}

Self-reporting of previously diagnosed CSSs from the CSI-BP part B was evaluated. This information was used to provide three subject categories with presumably three different levels of CS involvement. The 63 healthy controls were allocated to the "low CS-symptom" group. Patients with a single diagnosis (OA, FM, MPS, or CTTH) and no self-reported CSS diagnosis on the CSI-BP part B comprised the "medium CS-symptom" group. Patients with multiple diagnoses (OA, FM, MPS, and CTTH) and the addition of one or more previous CSS diagnoses on the CSI-BP part B comprised the "high CS-symptom" group. The seven self-reported CSS diagnoses on the CSI-BP part B were restless-leg syndrome, chronic fatigue syndrome, FM, temporomandibular joint disorder, migraine or tension headache, irritable bowel syndrome, and multiple chemical sensitivities. These three subject subgroups were then evaluated by total CSI scores categorized by five severity levels - subclinical, mild, moderate, severe, and extreme - as has been recommended previously. ${ }^{12}$

\section{Assessment of serum BDNF and CPM task \\ CPM variables}

The 77 subjects from the identified subsample previously detailed were submitted to the CPM task using the protocol of Tousignant-Laflamme et al, ${ }^{25}$ which allows evaluation of the function of the descending pain-modulatory system. By attempting to modify the descending pain-modulatory system, indications of CS can be determined. Diffuse noxious inhibitory control was induced by immersing the nondominant hand in cold water $\left(0^{\circ} \mathrm{C}-1^{\circ} \mathrm{C}\right)$ for 60 seconds. ${ }^{25,26}$ The cold-pressor test is an intense nociceptive stimulus used to elicit diffuse noxious inhibitory control (test stimulus) "pain inhibits pain", ${ }^{26,27}$ and it allows us to modify the endogenous pain-modulating system. To quantify the function of the descending pain-modulatory system, we evaluated 
pain intensity by quantitative sensory testing (QST) ${ }^{26,28} \mathrm{An}$ experimentally predetermined test was done to determine individual thermal pain thresholds (TPTs), defined here as a numeric pain score of 6/10, using QST administered over the dominant forearm (the one opposite the cold-pressor test). In a separate test, during the last 30 seconds of cold-water immersion, the TPT procedure was applied to the dominant forearm using a temperature individually predetermined to induce a $6 / 10$ pain rating during the pretesting sessions. A CPM score was determined by subtracting the mean pain scores during TPT testing: (changes on the numeric pain scale [0-1] during QST + cold-water minus) minus (the first TPTs to induce pain on the numeric pain scale [6/10] using QST). Negative values on the CPM score indicated that inhibitory $\mathrm{CPM}$ was achieved. If the subject did not report a reduction in pain or reported an increase in pain during the CPM task, the descending modulatory systems were considered to have failed to modulate the nociceptive response. For data analysis, patients were divided into two groups: CPM responders, who reported a decrease in pain in the second TPT test, and CPM nonresponders, who reported no decreases or increases in pain during the second TPT test (indicating CPM failure and evidence of CS).

\section{Serum BDNF}

Because the release of BDNF has been found to be associated with the induction and maintenance of $\mathrm{CS},{ }^{9}$ blood samples were collected early in the morning for each subject in the identified subsample groups detailed previously. The blood samples were obtained in plastic tubes and centrifuged for 10 minutes at $4,500 \mathrm{rpm}$ at $4{ }^{\circ} \mathrm{C}$. Serum was stored at $-80^{\circ} \mathrm{C}$ for further BDNF assay. Serum-mediator concentrations were determined using BDNF (Chemicon CYT306, lower detection limit $7.8 \mathrm{pg} / \mathrm{mL}$; EMD Millipore, Billerica, MA, USA) enzyme-linked immunosorbent-assay kits, according to the manufacturer's instructions.

\section{Assessment of potential confounders}

The physician who performed the physical evaluation assessed each subject's analgesic use, which was defined as an average amount of analgesics used per week during the previous month. For data analysis, analgesic use was included as a dichotomous variable (the use of analgesics fewer than 4 days per week or use more than 4 days per week). This approach was chosen because the use of rescue analgesics by patients with chronic pain often changes each week, depending on their levels of pain.

\section{Statistical analysis}

Internal consistency reliability was assessed using Cronbach's $a$ for the CSI-BP to compare with the English version. Testretest correlation to assess reproducibility was used, and the intraclass coefficient (two-way random, type absolute agreement) was calculated for examining test-retest reliability. ${ }^{29}$ For factor analysis, a principal component analysis was conducted using Promax rotation. To select item loading, 0.4 was considered the relevant factor cutoff, and thus when the loading was less than 0.4 the item was not retained. ${ }^{30}$ We also excluded factors with eigenvalues greater than one.

Criterion validity was evaluated using the relationship between total CSI-BP scores (categorized by severity level) and the no, low, and high CS-symptom groups. For this assessment, an ANOVA was followed by a Bonferroni test to adjust for multiple comparisons. Independent $\chi^{2}$ tests were used for categorical variables, such as the presence or absence of the self-reported diagnosis of part B of the CSI, and the chronic pain syndromes with CSS-relevant factors identified by the physician. Cramer's $V$ was used as a measure of effect size for $\chi^{2}$ tests. Convergent validity was evaluated by the Pearson's correlation coefficient between CSI-BP score and the BP-PCS.

To determine the profile of accuracy of the CSI-BP in distinguishing between chronic pain subjects and pain-free healthy control subjects, an area under the curve (AUC) analysis was performed with exact binomial $95 \%$ confidence intervals (CIs). CSI-BP scores used to examine the AUC were adjusted by the effect of age, gender, years of school, and history of psychiatric diagnosis in a multiple regression model.

To assess the capability of the CSI-BP to identify the level of CS-related symptoms (no, low, and high) among the subjects with specific pain syndromes and the painfree healthy control subsamples $(n=77)$, we constructed an adjusted index of CSI-BP, using a forward-stepwise regression to select variables to include in a hierarchical multiple regression. ${ }^{4}$ This model generated an adjusted score on the CSI-BP, which was used to assess the correlation between the CSI-BP and the BDNF. Student's $t$-test for the independent sample was used to compare changes on the self-reported numeric pain scale (0-10) during CPM tasks, which were used to classify subjects into CPM responders and CMP nonresponders. ${ }^{31}$ To estimate sample size, we used the ratio of the number of subjects to the number of items. ${ }^{32}$ For all statistical analyses, significance was set at $P<0.05$. Data were analyzed using SPSS version 22.0 (IBM, Armonk, NY, USA). 


\section{Results}

\section{Test-retest reliability of the CSI-BP}

Test-retest reliability was assessed in a group of female patients with FM $(n=20)$. The test-retest correlation for each single item was 0.84 (95\% CI 0.62-0.94), and the average measure was 0.91 (95\% CI $0.77-0.97, P<0.001$ for both), indicating a high level of reliability between administrations. All item-analysis correlations were found to be statistically significant.

\section{Score distributions of the CSI-BP}

The demographic characteristics and scores of the CSI-BP parts A and B are presented in Table 1. There was a disproportionate number of females $(n=248)$ in our sample. The mean scores of the CSI-BP for males were 37.38 (17.05) and for females 46.61 (17.15) $(t=-3.15, P<0.001]$. The mean score on the CSI-BP for the total subject sample, including the healthy group, was 45.35 (SD 17.4). The median was 46 , and the range was $32.5-58$.

In evaluating total scores on part A of the CSI-BP, the FM group was statistically distinguishable from the OA and MPS groups, and all three chronic pain groups were statistically distinguishable from the healthy group. The mean total score in the FM group was nearly double that of the healthy control group. The average scores on FM were equivalent to reporting that all 25 symptoms occur "sometimes" (Table 1 ). Assessment of the number of self-reported previous CSS diagnoses on part B of the CSI-BP revealed that the mean number of diagnoses in FM was 2.21 (2.21) and MPS 2.21 (2.08), both groups more than double that of the OA (0.64 [0.83]). The healthy subjects averaged less than one CSS diagnosis $(0.03[0.17])$.

\section{Internal consistency of the CSI-BP}

Cronbach's $\alpha$ was 0.91 , indicating that each of the items contributed similarly to the construct that it intended to measure.

\section{Confirmatory factor analysis of the CSI-BP}

A confirmatory factor analysis was performed to investigate whether the original English CSI study dimensionality and factor-loading pattern was like the Brazilian subject sample. The mean (SD) of each item and the standardized factor loadings, including the specific CSI-BP question items, contributing to factors with item loading higher than 0.4 are shown in Table 2. The factor analysis yielded four factors that accounted for $49.01 \%$ of the variance in the data set.

\section{Relationship between subject subgroups with different types of chronic musculoskeletal pain with self-reported levels of CS-related symptoms}

Table 3 presents three subject groups determined to have low, medium, and high CS symptoms. Approximately $80 \%$ of the low CS-symptom group (ie, the nonpatient comparison subjects) scored below 40 and about $50 \%$ scored below 30 . Of patients in the medium CS-symptom subgroup, defined as having only one of the four study diagnoses (FM, MPS, CTTH, and OA), 76\% scored above 40 . In the high CS-symptom group, $57.8 \%$ scored above 50 and $29.47 \%$ scored above 60. The CSI severity-level groupings corresponded well with CSI-score distributions from these different subject samples.

\section{Relationship between scores on the CSI-BP and pain-catastrophizing scale}

Moderate positive correlations were found between total scores on the CSI-BP and total scores, as well as subscale scores on the BP-PCS. The Pearson correlation of the CSI-BP with the helplessness BP-PCS subscale was $r=0.68$ (95\% CI $0.61-0.71$ ), with the magnification BP-PCS subscale $r=0.63$ (95\% CI 0.55-0.69), with the rumination BP-PCS subscale $r=0.62(95 \%$ CI $0.54-0.68)$, and with the BP-PCS total score $r=0.68(95 \%$ CI $0.61-0.74)$.

Table I Demographic variables and CSI-BP scores for validation $(n=285)$

\begin{tabular}{|c|c|c|c|c|c|c|}
\hline Variables & Healthy controls' $(n=63)$ & $O A^{2}(n=31)$ & $\operatorname{MPS}^{3}(n=65)$ & $\mathrm{CTTH}^{4}(\mathrm{n}=53)$ & $\operatorname{FM}^{5}(n=73)$ & $P$ \\
\hline Gender (female) & $26(69.8 \%)$ & $31(100 \%)$ & $54(88.5 \%)$ & $29(26.7 \%)$ & 54 (94.5\%) & $<0.001$ \\
\hline Age (years) & $38.38(14.34)$ & $67.03(8.24)$ & $43.31(\mid I .5 I)$ & $36.23(12.18)$ & $49.94(10.95)$ & $<0.001$ \\
\hline Formal education (years) & $12.14(4.48)$ & $15.93(5.60)$ & $13.93(5.01)$ & $12(4.48)$ & $15.5(5.32)$ & $<0.001$ \\
\hline Psychiatric disease, yes (\%) $)^{\S}$ & - & $9(29 \%)$ & $38(58.46 \%)$ & $17(56.7 \%)$ & $43(58.9 \%)$ & $<0.001$ \\
\hline Part B, CSI-BP (diagnoses) $)^{€}$ & $0.68(0.89)^{2-5}$ & $1.37(I .31)^{1,3-5}$ & $2.83(1.3 \mathrm{I})^{12}$ & $2.30(1.02)^{1,2}$ & $3.23(1.68)^{1,2}$ & $<0.001$ \\
\hline CSI-BP (score) $)^{\epsilon}$ & $37.14(15.01)^{5}$ & $39.53(16.48)^{5}$ & $43.13(15.53)^{5}$ & $46.13(15.83)^{5}$ & $58.30(14.56)^{1-4}$ & $<0.001$ \\
\hline
\end{tabular}

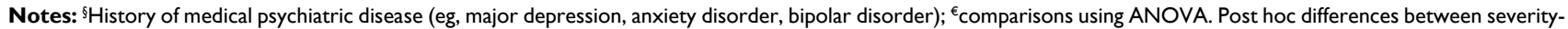
level groups are indicated via superscript numbers.

Abbreviations: CSI-BP, Central Sensitization Inventory - Brazilian population; OA, osteoarthritis; MPS, myofascial pain syndrome; CTTH, chronic tension-type headache; FM, fibromyalgia. 


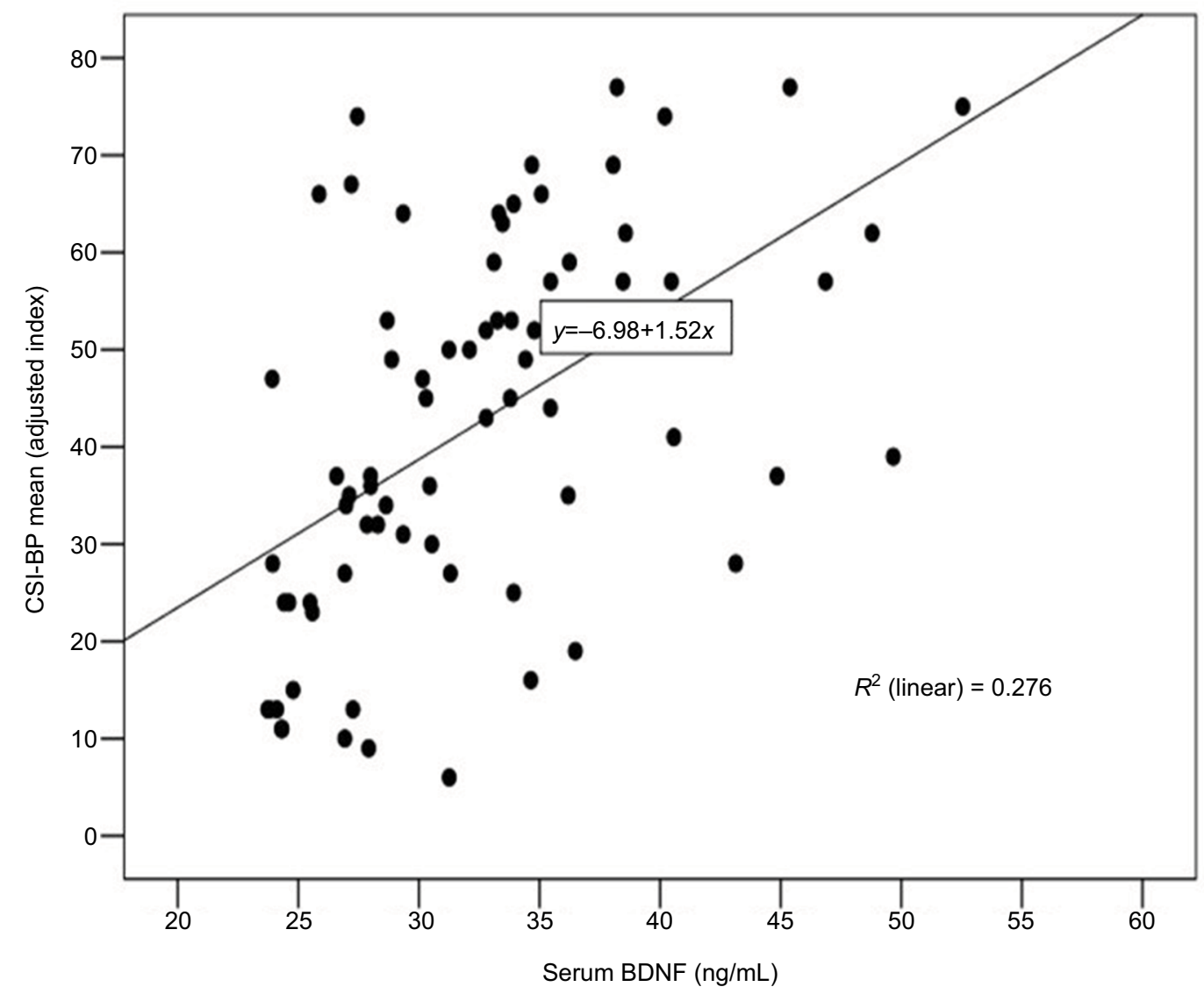

Figure 2 Scatter plots of serum BDNF and CSI-BP $(n=77)$.

Abbreviations: BDNF, brain-derived neurotrophic factor; CSI-BP, Central Sensitization Inventory - Brazilian population.

\section{Ability of the CSI-BP to discriminate between chronic pain and pain-free healthy control subjects}

The screening accuracy of the CSI-BP to discriminate between chronic pain subjects $(n=222)$ and healthy control subjects ( $\mathrm{n}=63$ ) was evaluated using CSI-BP scores adjusted by age, gender, years of school, and history of psychiatric diagnosis. These variables explained $27 \%$ of the proportion of the variance (fluctuation) in CSI-BP scores, which showed a profile of accuracy using a cutoff point of 35 related to sensitivity of 0.98 and specificity of 0.9 , with an AUC of 0.8 (95\% CI 0.76-0.86). These findings showed that according to this cutoff point, the CSI-BP classified correctly (ie, specificity) more than $90 \%$ of those that presented with CSS conditions.

\section{Ability of the CSI-BP to discriminate between subject subsamples}

The primary demographic variables and total CSI-BP scores for the four subsamples (healthy controls, OA, MPS, and FM) are presented in Table 4 . There were statistically significant differences among the four groups on self-reported pain and depressive symptoms, analgesic drug use, psychiatric history, blood levels of serum BDNF, previous CSS diagnoses on the CSI part B, and total CSI-BP scores.

To obtain the purer effect of group differences, CSI-BP scores were adjusted using a hierarchical multiple conditional regression analysis, as shown in Table 5. Level of education was negatively correlated with the CSI-BP score, while a higher level of depressive symptoms, greater number of diagnoses on part B of the CSI-BP, and higher pain scores were positively correlated with the CSI-BP. Therefore, CSIBP scores in this model allow us to assess on a standard scale if the CSI-BP is an index that is related to a serum marker of neuroplasticity, such as serum BDNF.

\section{Relationship between the CSI-BP and serum BDNF}

Total CSI-BP scores were correlated with serum levels of BDNF, a biological marker of neuroplasticity. The scatterplot of the raw CSI-BP and BDNF is presented in Figure 2. Pearson's correlation coefficient was 0.52 ( $95 \%$ CI $0.33-0.66$ ), and the coefficient of determination demonstrated that the proportion of variance explained by the association between 
Table 2 Factor analysis breakdown of the CSI-BP with the specific items that contributed to each factor $(n=285)$

\begin{tabular}{|c|c|c|c|c|c|c|}
\hline CSI-BP items & Mean (SD) & $\begin{array}{l}\text { Physical } \\
\text { symptoms }\end{array}$ & $\begin{array}{l}\text { Emotional } \\
\text { distress }\end{array}$ & $\begin{array}{l}\text { Headache/jaw } \\
\text { symptoms }\end{array}$ & $\begin{array}{l}\text { Urological } \\
\text { symptoms }\end{array}$ & $\begin{array}{l}\text { Items not } \\
\text { loading on } \\
\text { factors }\end{array}$ \\
\hline I. Unrefreshed in the morning & $2.39(1.18)$ & & 0.44 & & & \\
\hline 2. Muscles stiff/achy & $2.72(1.05)$ & 0.88 & & & & \\
\hline 3. Anxiety attacks & $2.12(1.13)$ & & 0.44 & & & \\
\hline 4. Grind clench/teeth & $1.67(1.43)$ & & & 0.5 & & \\
\hline 5. Diarrhea/constipation & $1.79(1.23)$ & & & & 0.47 & \\
\hline 6. Need help with daily activities & $\mathrm{I} .35(\mathrm{I} .4 \mathrm{I})$ & 0.59 & & & & \\
\hline 7. Sensitive to bright lights & $1.75(1.4 \mathrm{I})$ & & 0.5 & & & \\
\hline 8. Easily tired with physical activity & $2.39(1.28)$ & 0.73 & & & & \\
\hline 9. Pain all over body & $1.97(.137)$ & 0.77 & & & & \\
\hline 10. Headaches & $1.95(1.12)$ & & & 0.6 & & \\
\hline II. Bladder/urination pain & $0.69(1.01)$ & & & & 0.64 & \\
\hline 12. Do not sleep well & $2.17(1.18)$ & 0.49 & & & & \\
\hline 13. Difficulty concentrating & $2.09(1.09)$ & & 0.78 & & & \\
\hline 14. Skin problems & $1.74(1.42)$ & & & & & $x$ \\
\hline I5. Stress makes symptoms worse & $2.70(1.32)$ & & 0.5 & & & \\
\hline 16. Sad or depressed & $1.94(1.0)$ & & 0.67 & & & \\
\hline 17. Low energy & $2.13(1.10)$ & 0.46 & & & & \\
\hline 18. Tension, neck and shoulders & $2.80(1.15)$ & & & 0.63 & & \\
\hline 19. Pain in jaw & $0.55(1.00)$ & & & 0.48 & & \\
\hline 20. Certain smells make dizzy & $1.22(1.27)$ & & & 0.64 & & \\
\hline 21. Urinate frequently & $1.78(1.30)$ & & & & 0.63 & \\
\hline 22. Restless legs & $1.87(1.49)$ & 0.42 & & & & \\
\hline 23. Poor memory & $2.04(1.12)$ & & 0.72 & & & \\
\hline 24. Trauma as child & $1.21(1.34)$ & & 0.51 & & & \\
\hline 25. Pelvic pain & $1.26(1.35)$ & & & & 0.49 & \\
\hline
\end{tabular}

Notes: The following variance was found for each factor: factor I (physical symptoms) 32.81\%, factor 2 (headache/jaw symptoms) 5.36\%, factor 3 (emotional distress) $6.09 \%$, and factor 4 (urological symptoms) $4.94 \%$. The confirmatory factor analysis revealed that all items were significantly related to their specified factors, verifying the hypothesized relationships among the item and latent factors. It was noted that three items on the original English CSI did not load on any of the four factors, including items I (unrefreshed from sleeping), 5 (problems with diarrhea and/or constipation), and I4 (skin problems). With the CSI-BP, however, item I was retained in factor I and item 5 retained in factor 4 . Item 14 did not load any of the four factors. X, excluded item in the factorial analysis.

Abbreviations: CSI, Central Sensitization Inventory; CSI-BP, Central Sensitization Inventory - Brazilian population.

Table 3 CSI-BP severity-level scores of three subject subgroups, divided into five categories of severity $(n=285)$

\begin{tabular}{|c|c|c|c|c|c|c|c|c|}
\hline Subgroups & $\begin{array}{l}\text { Subclinical, } \\
0-29 \\
(n=53)\end{array}$ & $\begin{array}{l}\text { Mild, } \\
30-39 \\
(n=57)\end{array}$ & $\begin{array}{l}\text { Moderate, } \\
40-49 \\
(n=56)\end{array}$ & $\begin{array}{l}\text { Severe, } \\
50-59 \\
(n=56)\end{array}$ & $\begin{array}{l}\text { Extreme, } \\
50-59 \\
(n=62)\end{array}$ & $\chi^{2}$ & $P$-value & $\begin{array}{l}\text { Effect } \\
\text { size }\end{array}$ \\
\hline Low CS-symptom group $(n=63)^{\S}$ & $28(52.8 \%)$ & $15(26.3 \%)$ & $12(21.4 \%)$ & $4(7 \%)$ & $4(6.5 \%)$ & 38.3 & 0.001 & 0.28 \\
\hline Medium CS-symptom group $(n=49)^{*}$ & $16(30.2 \%)$ & $10(17.5 \%)$ & $12(21.4 \%)$ & $4(7 \%)$ & 7 (11.6\%) & & & 0.31 \\
\hline High CS-symptom group $(n=173)^{\pi}$ & $9(17 \%)$ & $32(56.1 \%)$ & $32(57.1 \%)$ & 49 (86\%) & $51(82.3 \%)$ & & & 0.16 \\
\hline
\end{tabular}

Notes: ${ }^{\S}$ Healthy control group; ${ }^{*}$ diagnosis of only one pain syndrome (OA/FM/MPS/CTTH); " ${ }^{\text {two }}$ or more diagnosed pain syndromes, plus one or more self-reported CSS on CSI-BP part B. Size effect interpreted as: small, 0.10-0.29; moderate, 0.30-0.49; and large, 0.5 or higher.

Abbreviations: CSI-BP, Central Sensitization Inventory-Brazilian population; CS, central sensitization; OA, osteoarthritis; FM, fibromyalgia; MPS, myofascial pain syndrome; CTTH, chronic tension-type headache; CSS, central sensitivity syndrome.

the CSI-BP and the BDNF was $27 \%\left(R^{2}=0.27\right)$. Increases in $\mathrm{BDNF}$ were associated with the higher CSI-BP scores.

\section{Relationship between the CSI-BP and responses to conditioned pain- modulation task}

The difference between the CSI-BP for CPM responders and CPM nonresponders is shown in Figure 3. The CPM nonresponders, who demonstrated dysfunction in the descending pain-modulatory system, had significantly higher CSI-BP scores than the CPM responders.

\section{Discussion}

The CSI-BP showed satisfactory psychometric evaluations, based on its internal consistency, construct validity, reproducibility convergent, discriminant validity, and factor structure. This showed that the translated version of CSI was stable over time, presenting excellent test-retest reliability, with a 
Table 4 Clinical and demographic characteristics among four subgroups $(n=77)$

\begin{tabular}{|c|c|c|c|c|c|}
\hline Characteristics & Healthy controls' $(n=I I)$ & $O A^{2}(n=26)$ & $\operatorname{MPS}^{3}(n=18)$ & $\operatorname{FM}^{4}(n=22)$ & $P$ \\
\hline$\overline{\text { Age (years) }}{ }^{€}$ & $40.09(10.93)^{2,4}$ & $67.5(8.08)^{2-4}$ & $48.66(10.14)^{2,4}$ & $53.72(6.97)^{1,2}$ & 0.001 \\
\hline Formal education (years) ${ }^{€}$ & $18.36(2.06)^{2-4}$ & $8.84(3.27)^{1,3}$ & $14.16(6.0)^{1,2,4}$ & $9.46(4.19)^{1,3}$ & 0.001 \\
\hline Number of chronic diseases & NA & $14(53.84 \%)$ & 8 (44.44\%) & $15(68.18 \%)$ & 0.21 \\
\hline Analgesic drugs used, yes ${ }^{t}$ & NA & $10(38.46 \%)$ & $14(77.77 \%)$ & $20(90.9 \%)$ & $<0.001$ \\
\hline Working, yes & 0 & $4(15.4 \%)$ & $3(16.7 \%)$ & $6(26.3 \%)$ & 0.13 \\
\hline History of psychiatric disease, yes & NA & 7 (26.9\%) & $9(50 \%)$ & $12(54.5 \%)$ & $<0.001$ \\
\hline $\operatorname{BDNF}(\mathrm{ng} / \mathrm{mL})^{\epsilon}$ & I6.I $(5.74)^{2-4}$ & $24.95(20.30)^{1,3}$ & $29.44(19.76)^{1,4}$ & $53.13(27.94)^{1-3}$ & $<0.001$ \\
\hline CPM responders (normal response) & II (I00\%) & 17 (64.5\%) & $13(72.2 \%)$ & $8(36.4 \%)$ & 0.04 \\
\hline Pain on $V A S^{\epsilon}$ & $1.89(1.24)^{2-4}$ & $6.61(1.89)^{\prime}$ & $6.90(2.67)^{\prime}$ & $6.80(1.70)^{\prime}$ & $<0.001$ \\
\hline Pain-pressure threshold $€$ & NA & $3.88(1.57)^{4}$ & $4.46(3.64)^{4}$ & $2.10(1.28)^{2,3}$ & $<0.001$ \\
\hline Beck Depression Inventory II ${ }^{€}$ & $3.28(2.09)^{2-4}$ & $11.61(8.13)^{1,3,4}$ & $17.67(10.94)^{1,2}$ & $17.00(6.11)^{1,2}$ & $<0.001$ \\
\hline Number of diagnoses, part B CSI-BP€ & $0.63(0.92)^{2-4}$ & $1.34(1.23)^{1,3,4}$ & $2.40(1.75)^{1,2}$ & $2.95(1.49)^{1,2}$ & $<0.001$ \\
\hline CSI-BP€ & $31.18(11.57)^{2-4}$ & $40.61(10.38)^{1,4}$ & $41.22(10.38)^{1,4}$ & $57.54(12.38)^{1-3}$ & $<0.001$ \\
\hline
\end{tabular}

Notes: Data presented as $\mathrm{n}(\%)$ or mean (SD). ${ }^{€}$ Comparisons using ANOVA. Post hoc differences of 0.05 among groups indicated via superscript numbers. ${ }^{\ddagger}$ Analgesic drugs used more than three times per week during the last 3 months. CPM responders who reported a decrease in pain in the second TPT test, and CPM nonresponders, who reported no decreases, or increases in pain, during the second TPT test (indicating CPM failure and evidence of CS).

Abbreviations: OA, osteoarthritis; MPS, myofascial pain syndrome; FM, fibromyalgia; NA, not applicable; BDNF, brain-derived neurotrophic factor; CPM, conditioned pain modulation; VAS, visual analogue scale; CSI-BP, Central Sensitization Inventory - Brazilian population; TPT, thermal pain threshold; CS, central sensitization.

Table 5 Hierarchical multiple conditional regression analysis to adjust CSI-BP score for potential confounders $(n=77)$

\begin{tabular}{llllll}
\hline & $\boldsymbol{V}$ & SE & $\boldsymbol{\beta}$ & $\boldsymbol{t}$ \\
\hline Years at school* & -1.087 & 0.395 & -0.303 & -2.75 & $\boldsymbol{P}$ \\
Beck Depression Inventory $^{\$}$ & 1.105 & 0.196 & 0.546 & 5.64 & 0.007 \\
Number of diagnoses, part B CSI-BP $^{*}$ & 3.548 & 1.155 & 0.301 & 3.07 & 0.003 \\
Visual analogue scale $^{\$}$ & 1.519 & 0.696 & 0.228 & 2.18 & 0.032 \\
\hline
\end{tabular}

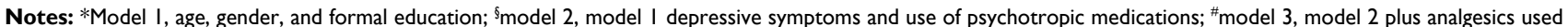
at least three times/week in last 3 months and pain scores on the visual analogue scale and number of diagnoses of part B of CSI-BP; ${ }^{\$}$ model 4 , model 3 plus type of chronic pain syndrome that could have been affected by all the variables studied in the previous hierarchical levels.

Abbreviations: CSI-BP, Central Sensitization Inventory - Brazilian population; SE, standard error.

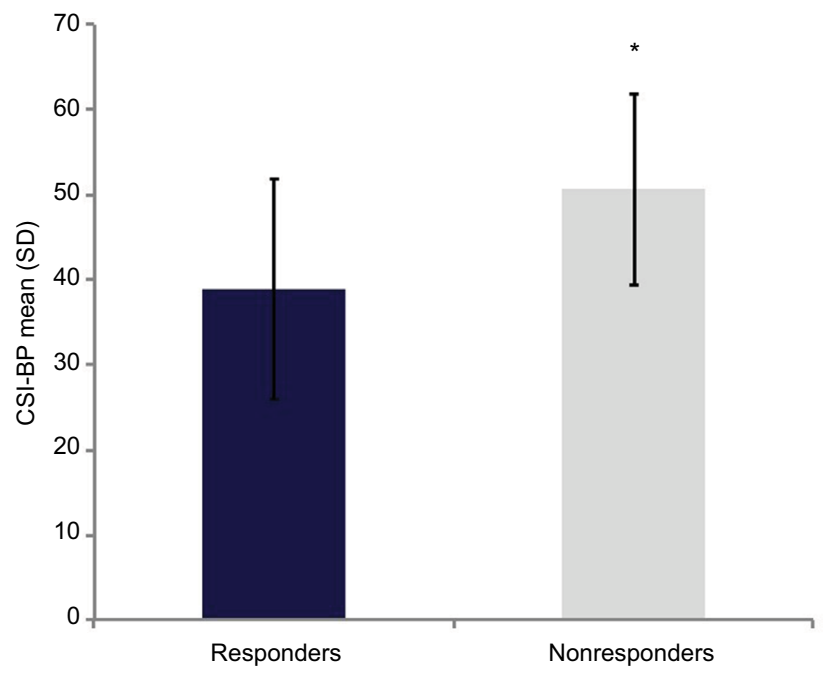

Figure 3 Comparisons of the CSI-BP between nonresponders (NPS ${ }_{0-10} \mathrm{HPTI}-$ HPTO $\geq 0, n=28$ ) and responders $\left(\mathrm{NPS}_{0-10}\right.$ HPTI - HPTO $\left.<0, n=49\right)$ (total $(n=77)$. Notes: *Differences between groups (responders and nonresponders to conditioned pain-modulation task) compared by $t$-test for independent samples. Error bars indicate standard deviation of the mean.

Abbreviations: CSI-BP, Central Sensitization Inventory - Brazilian population; HPT, heat pain threshold; NPS, numeric pain scale.

performance similar to that observed with the Dutch CSI. ${ }^{13}$ The moderate correlation coefficient of the CSI-BP with the
BP-PCS ${ }^{22}$ is a criterion usually considered satisfactory for establishing construct validity. ${ }^{33}$ Also, this finding suggests that catastrophizing pain has an overlap with the CS phenomenon, which leads to behavioral and emotional changes for people experiencing chronic pain. ${ }^{34}$ This hypothesis is supported by a moderate positive correlation of catastrophizing with intracortical facilitation measured via transcranial magnetic stimulation in patients with chronic MPS. ${ }^{35}$

The CSI-BP showed criterion-group validity to differentiate between subgroups. A cutoff of 35 found good sensitivity $(0.98)$ for correctly identifying patients and good specificity (0.87) for correctly identifying control subjects, which resulted in $80 \%$ of subjects being accurately identified. In addition to very good discrimination accuracy, this result is interesting, because a total score of 40 was previously determined to be the best cutoff for the English version of the CSI. The cutoff discrepancy between the English CSI and the CSI-BP is most probably explained by the different subject samples evaluated. Also, CSI-BP discriminatory properties were demonstrated to differentiate the mean among specific chronic pain conditions (FM, OA, CTTH, and MPS) and a healthy group. These results are underscored by a previous 
study in chronic pain and specialized clinic pain. ${ }^{36}$ Selection bias was possible, and as such the external validity of these results to other samples of chronic pain patients from different settings is uncertain, such as patients under the care of a family physician. Although our data are consistent with findings observed in the original version and adapted and published into various European, Asian, and South American languages, ${ }^{14-18}$ future research could evaluate the utility of the instrument in heterogeneous-sample subjects in diverse clinical settings.

Validity was also assessed with two objective biological markers of CS. The results of the CPM task categorized subjects into two groups. Subjects who showed that inhibitory CPM was achieved were classified as CPM responders. Subjects who showed a failure of the descending modulatory system to modulate the nociceptive response (indicating CS) were categorized as CPM nonresponders. According to a spectrum of responders and nonresponders to the CPM task, the current findings suggest a relationship between the descending modulatory system and subjects with presumably different levels of CS-related symptomatology, from patients with FM to healthy control subjects. The CPM task evaluated self-reported pain levels, but CS is not only associated with pain. It can also affect cognitive and emotional functioning in general. ${ }^{37}$ This statement is supported by similar dysfunction in the descending pain-modulatory system reported in lesions in brain regions implicated in descending pain modulation (ie, traumatic brain injury and multiple sclerosis), including the medial prefrontal cortex and rostral anterior cingulate cortex. ${ }^{38}$ Additionally, this dysfunction has been demonstrated when alterations occurred in the integrity of brain regions involved in both pain control and cognitive and emotional functioning. Thereby, changes in this network could explain the relationship between the severity of the self-reported CS-related symptoms on the CSI-BP and the dysfunction of the corticospinal pain-modulatory system measured by the CPM task. As is known, the activity of interneurons in the descending inhibitory system is mediated by the synthesis of neurotransmitters (GABA and glycine), serotonin, and norepinephrine. ${ }^{39}$ However, a lack of function in the descending pain-modulatory system as assessed by the CPM task suggests that the role of these neurobiological systems is weak, and a greater score on the CSI-BP in CPM nonresponders indicates higher dysfunction in the inhibitory descending network.

This is an interesting finding with potential applicability to help clinicians personalize the best therapeutic approach based on the level of CS-related symptomatology of each patient. Particularly intriguing is how CPM serves as a marker with a large size effect to identify impairment of the descending pain-modulatory system in populations with long-term pain conditions. ${ }^{40}$ In another way, this finding assesses a consequent downward negative spiral of pain induced by chronic pain. While these results need to be confirmed in further studies before allowing for a definitive conclusion, according to recent research in our laboratory, we demonstrated that the loss of descending pain inhibition was associated with an increase in intracortical facilitation measured by transcranial magnetic stimulation, higher serum BDNF, and a greater level of disability related to pain. ${ }^{41}$ Therefore, the relationship between the variation in CSI-BP and CPM scores could represent the imbalance in the neuroplasticity mediators involved in the modulation of the excitatory/inhibitory CNS. Therefore, a higher score on the CSI may be useful in helping to identify patients prone to severe chronic pain or those with a greater propensity to respond to certain treatments, such as demonstrated by duloxetine in treating neuropathic pain and OA. ${ }^{42,43}$ Future studies should investigate if CSI scores can be a useful tool for evaluating new approaches for improving the function of the neurobiological systems involved in CS and CSS.

The CSI-BP in the present study was also correlated with serum BDNF. There is compelling evidence that BDNF is a ubiquitous pain mediator at many levels of the nervous system and that serum levels are an indirect assessment of the neuroplasticity changes associated with CS. Accordingly, it has also been demonstrated that vascular circulating BDNF represents $70 \%-80 \%$ of that produced in the $\mathrm{CNS} .{ }^{44}$ Although the transport of BDNF produced in the CNS occurs through the blood-brain barrier via saturable systems, it has been widely demonstrated using different approaches with effect in the CNS that variations in the serum levels of this neurotrophic factor reflect the impact of such interventions. Included among such interventions are antidepressant drugs, electroconvulsive therapy, ${ }^{45}$ electroacupuncture, ${ }^{6}$ transcranial magnetic stimulation, ${ }^{46}$ and transcranial direct current stimulation. ${ }^{47}$ Therefore, this set of evidence shows that changes in peripheral BDNF levels are associated with changes involving a neuroplasticity process. As such, the moderate positive correlation between BDNF and the CSI-BP suggests that chronic pain can induce reorganization of the circuits involved in pain processing, which can at least in part be measured by changes in CSI-BP scores. Nevertheless, we should exercise parsimony in the interpretation of these results, because we can only indirectly infer if changes in BDNF are related to the severity of CS symptoms assessed by the CSI-BP. 
The present study shows that the CSI-BP is related to markers that comprise the neuroplasticity processes involved in CS-related symptoms (ie, BDNF). However, several potential limitations in this study need to be addressed. First, considering there was a nonrandom selection of patients, we cannot exclude some selection bias, although we had recruited subjects in a pain clinic from the primary units and by newspaper publicity. However, it is noteworthy that our results are supported by psychophysical and biomarkers less prone to possible bias. Second, our study presents an imbalance in the number of male to female subjects, which may influence gender differences in attitudes and health behavior. ${ }^{48} \mathrm{Also}$, we observed an imbalance in several characteristics between pain syndromes, which can affect CSI-BP score, biological BDNF secretion, and the function of the descending pain-modulatory system. ${ }^{32}$ Third, in a clinical study it is not possible to directly assess and isolate the effect of each of these potential confounding factors in BDNF secretion or in the descending painmodulatory system by CSI measures. Therefore, to generate a CSI-BP adjusted by the imbalance between the covariates, we constructed an adjusted index using a hierarchical regression model based on propensity-score calibration. ${ }^{49}$ The CSI-BP controlled for the potential concealed influence of these set of factors, allowing us to evaluate the relationship between CSI-BP score as the dependent variable and the BDNF and in the descending pain-modulatory system function on a standard scale ${ }^{46,50}$ Fourth, years of schooling showed a negative correlation with CSI-BP score. It is plausible that low literacy is associated with a variety of adverse health conditions and presents a barrier to effective care. The positive correlation between depressive symptoms and the CSI-BP may concern the cognitive, psychological, and behavioral changes observed in patients with chronic pain, ${ }^{51}$ since the depression itself is related to worse health status. Finally, the recommended CSI symptom-severity levels can provide a guideline for interpreting CSI scores in research trials and clinical decision making. ${ }^{13}$

This study provides evidence for the consistent psychometric properties of the CSI-BP. The CSI-BP showed good discriminative properties and associations with biological markers of CS, including serum BDNF and dysfunction of the descending pain-modulatory system. Therefore, these results suggest that the CSI-BP represents a valuable tool for use in scientific studies and in the clinical setting involving patients with chronic pain.

\section{Acknowledgments}

This research was supported by grants and material support from the following Brazilian agencies: Committee for the
Development of Higher Education Personnel (CAPES PNPD/CAPES) (grants to LCA) and material support; National Council for Scientific and Technological Development (CNPq) (grants to ILST and WC); Postgraduate Program in Medical Sciences at the School of Medicine of the Federal University of Rio Grande do Sul (material support); Postgraduate Research Group at the Hospital de Clínicas de Porto Alegre (FIPE-HCPA) (material support); and the Brazilian Innovation Agency (FINEP) (process number 1245/13; WC).

\section{Author contributions}

All authors made a significant contribution to study concept and design, acquisition of data, or analysis and interpretation of data, drafting or revising the manuscript for important intellectual content, and approval of the final version to be published.

\section{Disclosure}

The authors report no conflicts of interest in this work.

\section{References}

1. Fayaz A, Croft P, Langford RM, Donaldson LJ, Jones GT. Prevalence of chronic pain in the UK: a systematic review and meta-analysis of population studies. BMJ Open. 2016;6(6):e010364.

2. Miranda VS, Decarvalho VB, Machado LA, Dias JM. Prevalence of chronic musculoskeletal disorders in elderly Brazilians: a systematic review of the literature. BMC Musculoskelet Disord. 2012;13:82.

3. Schur EA, Afari N, Furberg H, et al. Feeling bad in more ways than one: comorbidity patterns of medically unexplained and psychiatric conditions. J Gen Intern Med. 2007;22(6):818-821.

4. Deitos A, Dussán-Sarria J, Souza A, et al. Clinical value of serum neuroplasticity mediators in identifying the central sensitivity syndrome in patients with chronic pain with and without structural pathology. Clin J Pain. 2015;31(11):959-967.

5. Yunus MB. Central sensitivity syndromes: a unified concept for fibromyalgia and other similar maladies. J Indian Rheum Assoc. 2000;8(1):27-33.

6. Ji RR, Kohno T, Moore KA, Woolf CJ. Central sensitization and LTP Do pain and memory share similar mechanisms? Trends Neurosci. 2003;26(12):696-705.

7. Woolf CJ, Salter MW. Neuronal plasticity: increasing the gain in pain. Science. 2000;288(5472):1765-1769.

8. Latremoliere A, Woolf CJ. Central sensitization: a generator of pain hypersensitivity by central neural plasticity. J Pain. 2009;10(9):895-926.

9. Trang T, Beggs S, Salter MW. Brain-derived neurotrophic factor from microglia: a molecular substrate for neuropathic pain. Neuron Glia Biol. 2011;7(1):99-108.

10. Nijs J, Torres-Cueco R, van Wilgen CP, et al. Applying modern pain neuroscience in clinical practice: criteria for the classification of central sensitization pain. Pain Physician. 2014;17(5):447-457.

11. Nijs J, Apeldoorn A, Hallegraeff H, et al. Low back pain: guidelines for the clinical classification of predominant neuropathic, nociceptive, or central sensitization pain. Pain Physician. 2015;18(3): E333-E346.

12. Neblett R, Hartzell MM, Mayer TG, Cohen H, Gatchel RJ. Establishing clinically relevant severity levels for the central sensitization inventory. Pain Pract. 2017;17(2):166-175. 
13. Kregel J, Vuijk PJ, Descheemaeker F, et al. The Dutch central sensitization inventory (CSI): factor analysis, discriminative power, and test-retest reliability. Clin J Pain. 2015;32(7):624-630.

14. Bid D, Neela S, Rathod PV, Ramalingam T. Content validity and testretest reliability of the Gujarati version of the central sensitization inventory. Natl J Integr Res Med. 2016;7(5):18-24.

15. Cuesta-Vargas AI, Roldan-Jimenez C, Neblett R, Gatchel RJ. Crosscultural adaptation and validity of the Spanish central sensitization inventory. SpringerPlus. 2016;5(1):1837.

16. Knezevic A, Neblett R, Jeremic-Knezevic M, Tomasevic-Todorovic S, Boskovic K, Cuesta-Vargas AI. Cross cultural adaptation and psychometric validation of the Serbian version of the Central Sensitization Inventory (CSI). Pain Pract. Epub August 4, 2017.

17. Kregel J, Vuijk PJ, Descheemaeker F, et al. The Dutch central sensitization inventory (CSI): factor analysis, discriminative power, and test-retest reliability. Clin J Pain. 2016;32(7):624-630.

18. Pitance L, Piraux E, Lannoy B, et al. Cross cultural adaptation, reliability and validity of the French version of the central sensitization inventory. Man Ther. 2016;25:e83-e84.

19. Jinks C, Jordan K, Croft P. Measuring the population impact of knee pain and disability with the Western Ontario and McMaster universities osteoarthritis index (WOMAC). Pain. 2002;100(1-2):55-64.

20. Olesen J. The International Classification of Headache Disorders: 2nd edition (ICHD-11). Rev Neurol (Paris). 2005;161(6-7):689-691.

21. Wolfe F, Häuser W. Fibromyalgia diagnosis and diagnostic criteria. Ann Med. 2011;43(7):495-502.

22. Sehn F, Chachamovich E, Vidor LP, et al. Cross-cultural adaptation and validation of the Brazilian Portuguese version of the pain catastrophizing scale. Pain Med. 2012;13(11):1425-1435.

23. Sullivan MJ, Bishop SR, Pivik J. The pain catastrophizing scale: development and validation. Psychol Assess. 1995;7(4):524-532.

24. Gomes-Oliveira MH, Gorenstein C, Neto FL, Andrade LH, Wang YP. Validation of the Brazilian Portuguese version of the Beck Depression Inventory-II in a community sample. Rev Bras Psiquiatr. 2012;34(4):389-394.

25. Tousignant-Laflamme Y, Pagé S, Goffaux P, Marchand S. An experimental model to measure excitatory and inhibitory pain mechanisms in humans. Brain Res. 2008;1230:73-79.

26. von Baeyer CL, Piira T, Chambers CT, Trapanotto M, Zeltzer LK. Guidelines for the cold pressor task as an experimental pain stimulus for use with children. J Pain. 2005;6(4):218-227.

27. Willer JC, De Broucker T, Le Bars D. Encoding of nociceptive thermal stimuli by diffuse noxious inhibitory controls in humans. $J$ Neurophysiol. 1989;62(5):1028-1038.

28. Schestatsky P, Stefani LC, Sanches PR, et al. Validation of a Brazilian quantitative sensory testing (QST) device for the diagnosis of small fiber neuropathies. Arq Neuropsiquiatr. 2011;69(6):943-948.

29. Shrout PE, Fleiss JL. Intraclass correlations: uses in assessing rater reliability. Psychol Bull. 1979;86(2):420-428.

30. Fayers PM, Machin D. Quality of Life: Assessment, Analysis and Interpretation. Chichester, UK: John Wiley \& Sons; 2000.

31. Botelho L, Morales-Quezada L, Rozisky J, et al. A framework for understanding the relationship between descending pain modulation, motor corticospinal, and neuroplasticity regulation systems in chronic myofascial pain. Front Hum Neurosci. 2016;10:308.

32. Rouquette A, Falissard B. Sample size requirements for the internal validation of psychiatric scales. Int JMethods Psychiatr Res. 2011;20(4):235-249.

33. Peterson RA. A meta-analysis of Cronbach's coefficient $\alpha$. J Consum Res. 1994;21(2):381-391.
34. Trivedi MH. The link between depression and physical symptoms. Prim Care Companion J Clin Psychiatry. 2004;6 Suppl 1:12-16.

35. Lumly MA, Cohen JL, Borszcz GS, et al. Pain and emotion: a biopsychosocial review of recent research. J Clin Psychol. 2011;67(9): 942-968.

36. Mayer TG, Neblett R, Cohen H, et al. The development and psychometric validation of the central sensitization inventory. Pain Pract. 2012;12(4):276-285.

37. Bushnell MC, Ceko M, Low LA. Cognitive and emotional control of pain and its disruption in chronic pain. Nat Rev Neurosci. 2013;14(7):502-511.

38. Pessoa L. On the relationship between emotion and cognition. Nat Rev Neurosci. 2008;9(2):148-158.

39. Lewis GN, Heales L, Rice DA, Rome K, McNair PJ. Reliability of the conditioned pain modulation paradigm to assess endogenous inhibitory pain pathways. Pain Res Manag. 2012;17(2):98-102.

40. Caumo W, Deitos A, Carvalho S, et al. Motor cortex excitability and BDNF levels in chronic musculoskeletal pain according to structural pathology. Front Hum Neurosci. 2016;10:357.

41. Yarnitsky D, Granot M, Nahman-Averbuch H, Khamaisi M, Granovsky Y. Conditioned pain modulation predicts duloxetine efficacy in painful diabetic neuropathy. Pain. 2012;153(6):1193-1198.

42. Wang ZY, Shi SY, Li SJ, et al. Efficacy and safety of duloxetine on osteoarthritis knee pain: a meta-analysis of randomized controlled trials. Pain Med. 2015;16(7):1373-1385.

43. Rasmussen P, Brassard P, Adser H, et al. Evidence for a release of brain-derived neurotrophic factor from the brain during exercise. Exp Physiol. 2009;94(10):1062-1069.

44. Brunoni AR, Lopes M, Fregni F. A systematic review and meta-analysis of clinical studies on major depression and BDNF levels: implications for the role of neuroplasticity in depression. Int $J$ Neuropsychopharmacol. 2008;11(8):1169-1180.

45. Brunoni AR, Baeken C, Machado-Vieira R, Gattaz WF, Vanderhasselt MA. BDNF blood levels after electroconvulsive therapy in patients with mood disorders: a systematic review and meta-analysis. World J Biol Psychiatry. 2014;15(5):411-418.

46. Dall'Agnol L, Medeiros LF, Torres IL, et al. Repetitive transcranial magnetic stimulation increases the corticospinal inhibition and the brain-derived neurotrophic factor in chronic myofascial pain syndrome: an explanatory double-blinded, randomized, sham-controlled trial. J Pain. 2014;15(8):845-855.

47. Brietzke A, Rozisky J, Dussan-Sarria J, et al. Neuroplastic effects of transcranial direct current stimulation on painful symptoms reduction in chronic hepatitis C: a phase II randomized, double blind, sham controlled trial. Front Neurosci. 2016;9:498.

48. Ray-Mazumder S. Role of gender, insurance status and culture in attitudes and health behavior in a US Chinese student population. Ethn Health. 2001;6(3-4):197-209.

49. Glynn RJ, Schneeweiss S, Stürmer T. Indications for propensity scores and review of their use in pharmacoepidemiology. Basic Clin Pharmacol Toxicol. 2006;98(3):253-259.

50. Stefani LC, Torres IL, de Souza IC, Rozisky JR, Fregni F, Caumo W. BDNF as an effect modifier for gender effects on pain thresholds in healthy subjects. Neurosci Lett. 2012;514(1):62-66.

51. Meeus M, Nijs J. Central sensitization: a biopsychosocial explanation for chronic widespread pain in patients with fibromyalgia and chronic fatigue syndrome. Clin Rheumatol. 2007;26(4):465-473. 


\section{Supplementary material \\ Phase I: translation, synthesis, and back- translation \\ Translation}

The original English version of the Central Sensitization Inventory (CSI) was translated into Brazilian Portuguese. Cross-cultural adaptation was carried out by previously published guidelines. ${ }^{1-3}$ Four native Brazilian Portuguese speakers carried out independent translations of the paincatastrophizing scale from English to Brazilian Portuguese: a professional translator, a psychologist, a physician with pain specialization, and a linguist. Forward-translations were compared with one another and with the original English version. After discussing any discrepancies, the four versions were combined into one Brazilian Portuguese version.

Several items were difficult to translate between English and Brazilian Portuguese. For item 3 (anxiety attacks), we decided to use the term "crises". For item 11, "discomfort in the bladder" was modified to "discomfort and burning sensation". For item 25, "trauma" was amended to "suffering", because "trauma" in Brazilian Portuguese is more associated with physical lesions.

\section{Back-translation}

Two native English speakers translated the original CSI to Brazilian Portuguese and also carried out a back-translation into English. Brazilian Portuguese was their second language. According to the definition of Deyo, ${ }^{4}$ both back-translators were considered bilingual, but they were not familiar with the subject matter of the questionnaire. Any gross inconsistencies or conceptual errors in the content of the translated versions in preparation for the expert committee meeting were corrected by a third bilingual person.

\section{Delphi method to assess semantics and conceptual content of each item}

A team of experts assessed each translated CSI item to ensure content validity. The expert committee consisted of one methodologist, one clinical research scientist, and all of the translators and back-translators. This expert panel was responsible for ensuring semantic and idiomatic equivalence and experiential and conceptual equivalence (ie, to address any peculiarities unique to the cultures examined) between the Brazilian Portuguese and English versions of the questionnaire. The committee members and the panel director corresponded by electronic communication. The panel director processed CSI adapted for a Brazilian population (CSI-BP) item information from the committee members and filtered out the relevant content until everyone came to a consensus on the content of each item on the CSI. If a consensus could not be reached on specific items, rounds coordinated by two clinical research scientists with experience in validating instruments were made until they came to a final consensus. ${ }^{5}$ All changes on the CSI-BP items were based on the consensus of members involved in the translation process. Their goal was to assure that each CSI item on the English and Brazilian versions referred to the same underlying concepts and had the same meanings, to produce the best idiomatic and conceptual (rather than merely literal) equivalence. The forward-translations were compared with one another and with the original English version. The forward-translations were compared with the original English version. As a result of this process, a pilot version of the CSI-BP was completed.

\section{Phase II: pretesting of CSI-BP \\ Assessment of CSI-BP in the pilot version}

Thirty medical school employees - half men, half women who work with chronic pain patients volunteered to evaluate the meaning of the translated questions and the layout of the "prefinal" version of the CSI-BP. In addition, they were interviewed to explore how they understood each of the 25 items. They had an average age of 30.67 (8.89 SD) years and an average of 19.56 formal years of schooling. Each employee's self-reported comprehension of the items was assessed with a $10 \mathrm{~cm}$ visual analogue scale (VAS; from 0 representing unclear to 10 representing entirely clear). The global mean \pm SD of comprehension of the 25 questions of CSI was $9.42 \pm 1.23$.

In addition to the medical school employees, 20 females with fibromyalgia volunteered to evaluate the meaning of the translated CSI-BP questions using the same VAS and interview process as the medical school employees. Their mean \pm SD for number of years of formal schooling was $10.41 \pm 4.35$, and their mean age was $50.77 \pm 9.88$ years. The global mean \pm SD of comprehension of the 25 questions of CSI-BP reported on the VAS was 8.01 \pm 1.21 .

All feedback from these subjects was evaluated by the translation workgroup (to assess face validity). Based on subject feedback, two questions were slightly modified to achieve the final Brazilian Portuguese version of the CSIBP. The final version of the CSI-BP is presented at http:// dorneuromodulacao.com.br. 


\section{References}

1. Beaton DE, Bombardier C, Guillemin F, Ferraz MB. Guidelines for the process of cross-cultural adaptation of self-report measures. Spine (Phila Pa 1976). 2000;25(24):3186-3191.

2. Guillemin F, Bombardier C, Beaton D. Cross-cultural adaptation of health-related quality of life measures: literature review and proposed guidelines. J Clin Epidemiol. 1993;46(12):1417-1432.
3. Wagner AK, Gandek B, Aaronson NK, et al. Cross-cultural comparisons of the content of SF-36 translations across 10 countries: results from the IQOLA project. $J$ Clin Epidemiol. 1998;51(11):925-932.

4. Deyo RA. Pitfalls in measuring the health status of Mexican Americans: comparative validity of the English and Spanish sickness impact profile. Am J Public Health. 1984;74(6):569-573.

5. Hsu C, Sandford B. The Delphi technique: making sense of consensus. Pract Assess Res Eval. 2007;12(10):1-8.

\section{Publish your work in this journal}

The Journal of Pain Research is an international, peer reviewed, open access, online journal that welcomes laboratory and clinical findings in the fields of pain research and the prevention and management of pain. Original research, reviews, symposium reports, hypothesis formation and commentaries are all considered for publication.
Dovepress

The manuscript management system is completely online and includes a very quick and fair peer-review system, which is all easy to use. Visit http://www.dovepress.com/testimonials.php to read real quotes from published authors. 